\title{
Cross-species hybridization of foot-and-mouth disease virus-infected BHK-21 cells using human and mouse oligonucleotide microarrays
}

\author{
H. ZHANG ${ }^{1,2}$, Q. WANG ${ }^{3}$, L. HAN ${ }^{1}$, C. ZHENG ${ }^{1}$, CH. SHEN ${ }^{1}$
}

\begin{abstract}
${ }^{1}$ State Key Laboratory of Virology, College of Life Sciences, Wuhan University, Wuhan, Hubei 430072, P. R. China; ${ }^{2}$ Bioengineering Department, Wuhan Bioengineering Institute, Luoyang Economy Development Zone, Wuhan, Hubei 430415, P. R. China; ${ }^{3}$ Center for Computational and Integrative Biology, Massachusetts General Hospital, Harvard Medical School, Boston, MA 02114, USA
\end{abstract}

Received August 8, 2016; revised December 19, 2016; accepted June 27, 2017

\begin{abstract}
Summary. - Foot-and-mouth disease virus (FMDV) has a dual capacity to induce either acute or persistent infection in host animals. Establishment of an in vitro cell model of FMDV persistent infection facilitates the study of the mechanism underlying this type of infection. In this study, we analyzed gene expression profiles of both acute and persistent infections using cross-species microarrays. Our data suggest that human microarrays are more efficient than mouse microarrays in hybridization with cDNA from BHK-21 cells although the mouse is closer to the Syrian hamster in taxonomy. A set of differentially expressed genes (DEGs) that may be involved in the determination of acute or persistent infection was identified by using human or mouse microarrays. Seven common DEGs were found in both human and mouse arrays and showed similar fold changes. Among the DEGs, 33 genes were selected for further validation by using qRT-PCR and presented consistent results. The analysis of Gene Ontology Biological Processes indicated that various biosynthetic and metabolic processes were negatively regulated in the group of acute infection whereas multicellular organismal development processes were positively regulated in the group of persistent infection. Our study demonstrates the plausibility and utility of using cross-species microarrays to study FMDV-infected mammalian cells. The combined use of two types of microarrays can be more informative in exploring the mechanisms underlying the infections of FMDV.
\end{abstract}

Keywords: foot-and-mouth disease virus; BHK-21; microarray; cross-species hybridization; acute infection; persistent infection

\section{Introduction}

Foot-and-mouth disease virus (FMDV) is the type species of the Aphthovirus genus within the Picornaviridae family. Infection of FMDV causes a highly contagious and economically important disease in cloven-hoofed animals, including cattle, pigs, sheep and goats; this disease is characterized by vesicular lesions in the mouth and on the feet, teats and nares (Sen and Saha, 1994; Brown, 1999; Balamurugan et al.,

*Corresponding author. E-mail: shenchao@whu.edu.cn; phone: +86-27-68754001.

Abbreviations: BHK-21 = Baby hamster kidney 21; $\mathrm{CSH}=$ crossspecies hybridization; DEGs = differentially expressed genes; $\mathrm{FMDV}=$ foot-and-mouth disease virus; GO:BP = Gene Ontology Biological Processes; qPCR = quantitative PCR
2004; Grubman and Baxt, 2004; Arzt et al., 2011). FMDV has a dual capacity to induce either an acute infection in clovenhooved animals, or an inapparent, persistent infection in ruminants (Brown, 1999; Grubman and Baxt, 2004; Mahy, 2005). FMDV usually becomes immediately cytopathic to the cultured cells, in which it can also establish a long-term persistent infection (de la Torre et al., 1985; Huang et al., 2011).

To facilitate the investigation of the mechanisms underlying FMDV persistence, an in vitro model of persistent infection was established with a biological clone of FMDV as well as cloned BHK-21 cells (Clarke, 1983; de la Torre et al., 1985, 1988; Cai et al., 2013). A remarkable feature of FMDV persistence in BHK-21 cells is the coevolution of cell-virus, in which the cells become gradually more resistant to FMDV, which in turn becomes more virulent to the 
parental BHK-21 cells ( de la Torre et al., 1988, 1989; Martin Hernandez et al., 1994; Herrera et al., 2008). Although genetic heterogeneity and phenotypic flexibility of FMDV in persistence have been well documented, it is recognized that it is the cell, instead of the virus, that plays a critical role in the initiation of persistence (Martin Hernandez et al., 1994; Zhang et al., 2013).

A microarray technique has been extensively used to study the changes in gene expression profiles in numerous cases. Unfortunately, the gene array of Syrian hamster is currently not available since the whole genome of Syrian hamster is unknown, which greatly hampers investigators to study the gene expression in the BHK-21 cells with FMDV persistence using a microarray technique. Recently, our lab first used the commercial human whole-genome oligo microarray on BHK-21 cell samples, and demonstrated the approach of cross-species hybridization (CSH) was actually feasible (Huang et al., 2011; Zhang et al., 2013). Plenty of information was derived from human microarray data (Zhang et al., 2013) and some of the new information from the present study may be even more valuable in elucidating the mechanism underlying FMDV infection.

There have been some issues regarding the cross-species hybridization (CSH) approach (Nieto-Diaz et al., 2007). $\mathrm{CSH}$ has been considered as a non-standard application of microarrays (Adjaye et al., 2004). It is known that the probes used for most of arrays are designed according to the sequences from one species, therefore, these probes may not exactly match the target cDNA derived from other species at many different nucleotide positions (Renn et al., 2004; Bar-Or et al., 2006). To overcome the inherently low signal of CSH, comparative cross-species hybridization was adopted. In $\mathrm{CSH}$, the performance of RNA isolated from the target species hamster was compared between two different reference species (human and mouse). This method had been taken by many researchers and the feasibility was also clarified (Rifkin et al., 2003; Held et al., 2004; Ji et al., 2004; Nagpal et al., 2004; Nuzhdin et al., 2004; Saetre et al., 2004; Brodsky et al., 2005; Chalmers et al., 2005; Gilad et al., 2005; Nowrousian et al., 2005; Neufeld et al., 2006; Vallee et al., 2006; Walker et al., 2006).

Syrian hamster (Mesocricetus auratus) belongs to the same rodent family as mouse. It is postulated that genes in Syrian hamster, compared with human ones, might be more conserved with those in mouse. Thus, the use of mouse arrays may reveal more information related to changes in the gene expression in Baby hamster kidney (BHK-21) cells, compared to the use of human arrays. In the present study, we applied these two types of arrays to investigate the gene expression profiles in BHK-21 cells and to evaluate whether mouse arrays were able to provide more information than human ones. A combined use of two types of arrays was expected to be more informative than the use of either type alone, and can also be very helpful in providing more information regarding the mechanistic differences between acute and persistent infections of FMDV in the mammalian cells such as BHK-21.

\section{Materials and Methods}

Cells and viruses. Virus strains of serotype O FMDV were obtained from Lanzhou Veterinary Research Institute, Chinese Academy of Agriculture Sciences. BHK-21 cells were provided by China Center for Type Culture Collection (CCTCC). Persistently infected BHK-21 cell lines were provided by Dr. Huang. In acute infection, cells were infected with FMDV at MOI of $3 \times 10^{-7}$ (lower titers of virus induced more stable response of infection). In persistent infection, persistently infected BHK-21 after 57 passages (PI57) were used for microarray and qRT-PCR analysis. Cells were cultured in Minimum Essential Medium (MEM, GiBCO, USA) supplemented with $10 \%$ heat-inactivated fetal bovine serum (FBS) (GiBCO, USA) at $37^{\circ} \mathrm{C}$ with $5 \% \mathrm{CO}_{2}$.

Virus infection and RNA extraction. Total RNA was extracted from acutely and persistently infected BHK-21 cells, respectively, using Trizol reagent (Life Technologies, Carlsbad, USA) according to the manufacturer's instructions. RNA was purified with RNeasy Mini Kit (Qiagen p/n 74104) and treated with baseline-ZERO DNase (EPICENTRE, Cat. No. DB0711K). The RNA quality was evaluated by agarose gel electrophoresis, and the quantity was determined by spectrophotometer (Eppendorf).

Microarray hybridization. One microgram of purified RNA was used for CDNA synthesis prior to hybridization to human or mouse whole-genome arrays (Agilent $4 \mathrm{X} 44 \mathrm{~K}$ ). The cDNA from uninfected BHK-21 cells was labeled with Cy3-dUTP, while the cDNA from FMDV acutely or persistently infected BHK-21 cells was labeled with Cy5-dUTP using the Agilent low RNA input linear amplification kit. Hybridization was performed overnight at $65^{\circ} \mathrm{C}$ with human or mouse whole-genome arrays (Agilent $4 \mathrm{X} 44$ K) using Agilent Gene Expression Hybridization Kit (Agilent p/n 5188-5242). Arrays were washed and scanned using an Agilent scanner (Agilent Technologies, Santa Clara, CA).

Data analysis. Agilent Feature Extraction software (version 11.0.1.1) was used to analyze array images and normalize the raw data with Lowess method. The data was subsequently processed using the GeneSpring GX v12.0 software package (Agilent Technologies). After data normalization, genes with the ratio of fluorescence signal intensity $\geq 1$ (the value from virus-infected samples divided by the value from uninfected samples) were selected for further analysis. Differentially expressed genes were identified through fold change filtering. Gene functional clustering was conducted with DAVID online bioinformatics resources 6.7 (Huang da et al., 2009a; Huang da et al., 2009b).

Real-time quantitative RT-PCR ( $q R T-P C R)$. The reaction of reverse transcription for CDNA synthesis included the following components: $1 \mu \mathrm{g}$ of total RNA, $1 \mu \mathrm{g}$ of Oligo (dT) 15 primers, $4 \mu \mathrm{l} 5 \times \mathrm{RT}$ 
buffer, $2 \mu \mathrm{l} 10 \mathrm{mmol} / \mathrm{l} \mathrm{dNTP}$ mix, $40 \mathrm{U}$ RNasin and $200 \mathrm{U}$ moloney murine leukemia virus (MMLV) reverse transcriptase (Promega). Real-time quantitative PCR was performed using SYBR green dye (Invitrogen) and Premix Taq (Promega). Primers were designed by using software Primer Premier 5. The sequences of primers are listed in Table S4. A two-step PCR $\left(15 \mathrm{~s}\right.$ at $95^{\circ} \mathrm{C}$ and $60 \mathrm{~s}$ at $\left.60^{\circ} \mathrm{C}\right)$ was performed with 40 cycles after an initial activation step $\left(95^{\circ} \mathrm{C}\right.$ for $10 \mathrm{~min}$ ) using the CFX96TM real-time PCR detection system (BIO-RAD). The melting curves from $60^{\circ} \mathrm{C}$ to $90^{\circ} \mathrm{C}$ were used to generate the standard melting curve.

\section{Results}

An overall comparison was performed between human and mouse microarrays

Human and mouse arrays were applied to evaluate the gene expression profiles in the FMDV-infected (acutely and persistently) and uninfected BHK-21 cells (used for normalization). There were about 41,000 probes in both human and mouse microarrays. Each probe was a 60-mer oligonucleotide with appropriate sensitivity and specificity for hybridization.

MA Plot method was used to perform an overall evaluation on human and mouse two-channel microarrays. In each array, acute and persistent infections were also analyzed comparatively. There were obvious differences between human and mouse microarrays in terms of the signal intensity (Fig. 1). Surprisingly, there were more MA points in human arrays than those in mouse arrays, indicating that human arrays hybridized more cDNAs of Syrian hamster than mouse arrays. In human arrays, the signal intensity in persistent infection was stronger than that in acute infection (Fig. 1a), while in mouse arrays, the signal intensity in two types of infections was almost the same (Fig. 1b). These results indicated that mouse microarray was less informative than the human microarray in hybridization with cDNA from

(a)

Human Microarrays
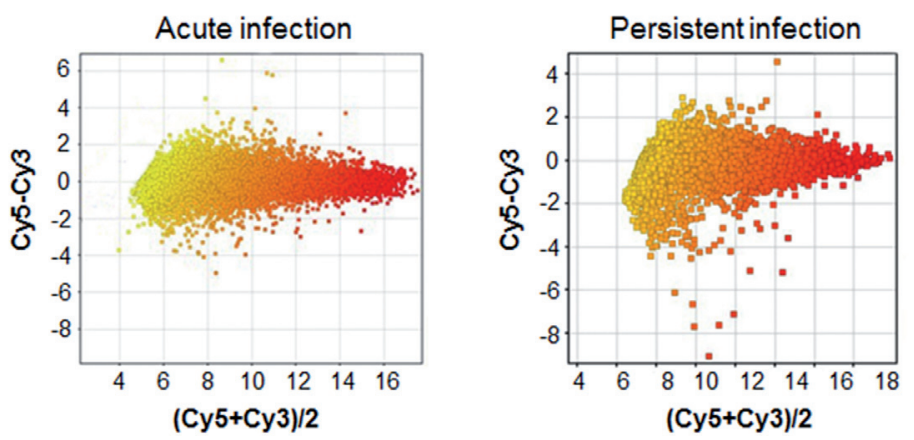

(b)

Mouse Microarrays
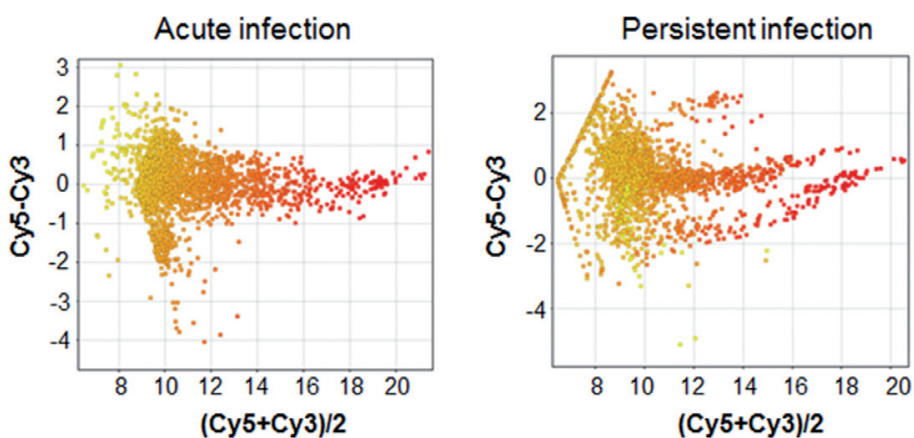

Fig. 1

Two-channel human and mouse microarrays were used to evaluate the signal intensity in acute and persistent infections

The MA Plot was used to assess the difference vs. the average of probes between two samples and also used in the two-color arrays to assess the relation between Cy3 (uninfected BHK-21 cell samples) and Cy5 (virus-infected BHK-21 cell samples) channels of the hybridization. The $\mathrm{x}$-axis represents the average $\log _{2}$ ratio of two channels, while the $y$-axis represents the difference of $\log _{2}$ ratio between two channels. The left panels of Fig. 1a and $1 \mathrm{~b}$ represent acute infection while the right panels represent persistent infection. (a) Signal intensity of human microarrays in both acute and persistent infection. (b) Signal intensity of mouse microarrays in both acute and persistent infection. 
Table 1. Number of hybridized probes and differently expressed genes in human and mouse arrays

\begin{tabular}{lccccc}
\hline & \multicolumn{2}{c}{ Acute infection } & & \multicolumn{2}{c}{ Persistent infection } \\
\cline { 2 - 3 } \cline { 5 - 6 } & Human & Mouse & & Human & Mouse \\
\hline Detected genes & 4618 & 1794 & & 4618 & 1794 \\
DEGs & 370 & 269 & & 506 & 483 \\
\hline
\end{tabular}

A fold change filtering was performed to identify significant differentially expressed genes between the two samples. The $\log _{2}$ ratio of fold changes $>=1$ or $\leq-1$ was set as the threshold.

BHK-21 cells, although the mouse is closer to the Syrian hamster than human in taxonomy. These observations were consistent with previous studies, in which human and mouse microarrays were used to hybridize woodchuck samples (Wang et al., 2009).

Differentially expressed genes (DEGs) were thoroughly compared between human and mouse microarrays

The results from the human and mouse arrays were obtained from four samples in this study. In both groups of acute and persistent infections, human microarrays detected 4618 genes while mouse ones merely detected 1794 genes (Table 1 and Fig. 1), indicating that human microarrays were much more efficient than mouse microarrays in these conditions. However, there was no significant difference between human and mouse arrays in terms of the DEG numbers although human arrays also detected more DEGs than mouse arrays in these two types of infections (Table 1).

We then analyzed fold change ( $\log _{2}$ ratio) distribution in DEGs. Human and mouse microarrays had similar distribu- tion trends in both acute and persistent infections (Fig. 2). The fold changes ( $\log _{2}$ ratio) of most of DEGs in both human and mouse arrays ranged from -2 to 2 (Fig. 2). In the range from -10 to -2 , both human and mouse microarrays detected 84 DEGs in persistent infection, whereas in the acute infection, the human arrays detected 19 DEGs while the mouse ones merely detected 9 DEGs. In the range from 2 to 10 , human arrays detected 11 DEGs in acute infection and 17 DEGs in persistent infection, while mouse arrays detected 25 DEGs in acute infection and 37 DEGs in persistent infection, indicating that mouse arrays were able to identify more differentially expressed genes than human arrays although they detected fewer genes in total.

\section{Significant DEGs were found in human and mouse} arrays

It is generally recognized that genes with large fold-change are of great interest to investigators. Fold changes $\geq 4$ or $\leq$ 0.25 were defined as the threshold for significant DEGs in this study. Some of the most significant DEGs found in human and mouse microarrays were summarized in Table S1 and Table S2.

In the group of acute infection, many classes of genes were identified in both human and mouse arrays, including protein phosphorylation genes such as PIK3R4 (0.01-fold, Table S1) and LRRK1 (0.20-fold, Table S2), translation-related genes such as RPL37 (4.44-fold, Table S1) and WBSCR22 (0.14-fold, Table S2), and transcription-related genes such as DEDD (6.77-fold, Table S1) and ERC1 (14.42-fold, Table S2). Notably, some unique classes of genes, including ubiquitin cycle-related genes such as RNF139 (0.18-fold, Table S1) and KPTN (4.63-fold, Table S1), and virus-response genes such (a)

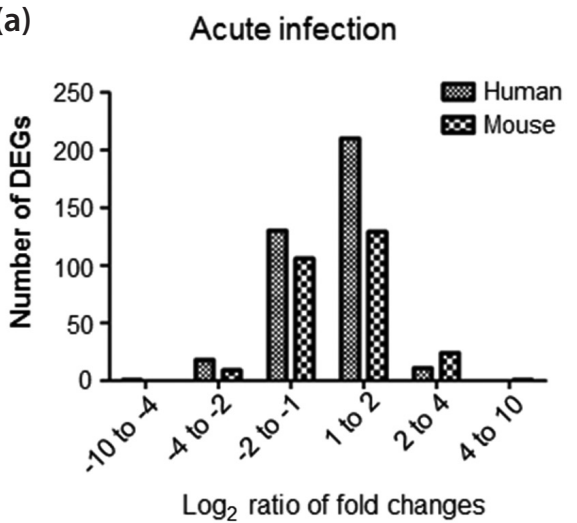

(b)

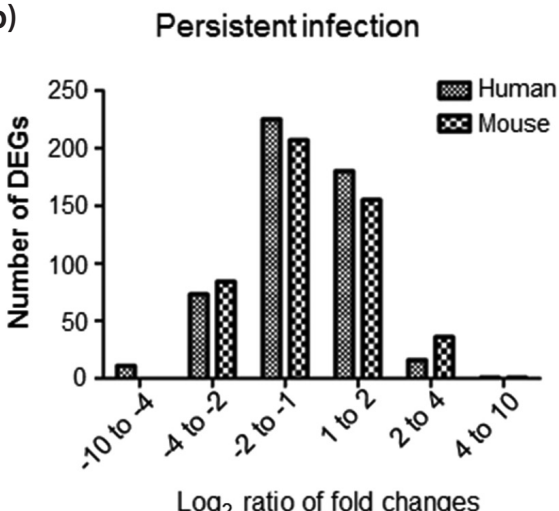

Fig. 2

Differentially expressed genes (DEGs) with distinct fold changes were identified by using human and mouse microarrays in both acute and persistent infections

(a) The comparison of DEGs' distribution between human and mouse microarrays in the condition of acute infection. (b) The comparison of DEGs distribution between human and mouse microarrays in the condition of persistent infection. The fold changes are presented in a log ${ }_{2}$ ratio ( $\mathrm{x}$-axis). 
(a)

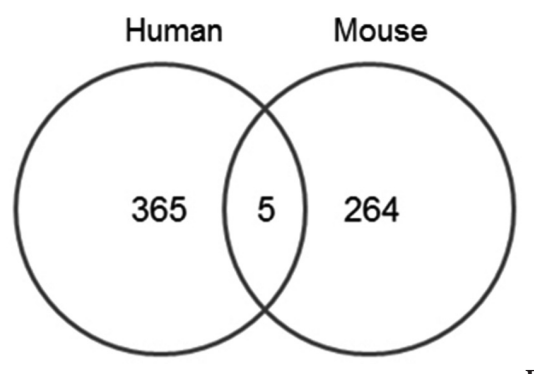

(b) Persistentinfection

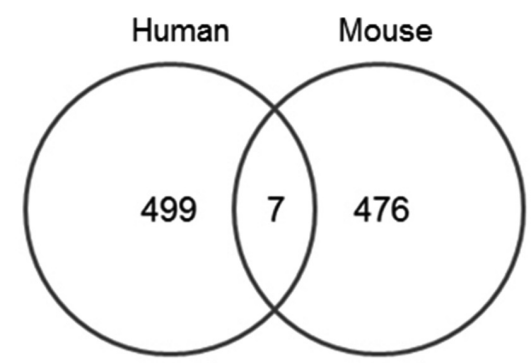

Fig. 3

Co-DEGs were identified in human and mouse microarrays

(a) The co-DEGs in acute infection were identified in human and mouse microarrays. (b) The co-DEGs in persistent infection were identified in human and mouse microarrays.

as DDX58 (5.62-fold, Table S1), were identified in human arrays while some other unique classes of genes, including exocytosis-related genes such as RIMS3 ( 0.14 fold, Table S2), and immune genes such as CD68 (8.17-fold, Table S2), were identified in mouse arrays.

In the group of persistent infection, genes involved in organismal growth and development, such as COL1A1 (0.01-fold, Table S1), SERPINF1 (0.05-fold, Table S1) and COL1A2 (24.45-fold, Table S1), were mostly identified in human microarrays. Interestingly, COL1A1 and COL1A2 had the opposite fold change although they are two forms of collagen type I, and both are involved in skeletal system development. Some genes that may play important roles in the persistence of FMDV such as CHAC1 (5.15-fold, Table S1), FOS (5.64-fold, Table S1), CREB5 (0.10-fold, Table S2), ITSN1 (0.11-fold, Table S2) and RGS16 (6.54-fold, Table S2) were identified as well. The calcium-dependent cellcell adhesion gene CDH11 (0.002-fold, Table S1), identified by using human microarray, was largely down-regulated in persistent infection. This might partially explain why persistently infected cells looked rounder and more scattered than acutely infected cells and normal BHK-21 cells (Huang et al., 2011; Zhang et al., 2013). The mitotic cytokinesis-related gene STAMBP (30.16-fold, Table S2), identified by using mouse microarray, was largely up-regulated in persistent infection. The up-regulation of this gene might contribute to the survival of the host cells after the lytic infection with FMDV.

Co-DEGs (common DEGs) were identified in human and mouse microarrays

Data analysis showed that 12 genes were commonly identified in human and mouse arrays, with 5 in the acute infection (Fig. 3a) and 7 in the persistent infection (Fig. 3b). In particular, there were seven genes that were found to be differentially expressed and had similar fold changes in both human and mouse arrays (Table 2). In acute infection, co-DEGs were related to DNA repair (Apex2), transcription (Mllt3) and signal transduction (Tle1). In persistent infection, co-DEGs were involved in organismal development (Sema4c), carbohydrate metabolic process (Ttn) and ubiquitin-dependent protein catabolic process (Usp11).

We then analyzed common GO biological processes (GO: $\mathrm{BP}$ ) in human and mouse microarrays by using DAVID bioinformatics resources online tool (Huang da et al., 2009a; Huang da et al., 2009b). The 370 and 269 DEGs of human and mouse arrays in the acute infection group, as well as 506 and 483 DEGs in the persistent infection group were mapped to GOTERM_BP_FAT category. As a result, only up-regulated GO biological processes were found in either human or mouse arrays (Table 3 ). In acute infection, the bioactivity of host cells was clearly compromised. Gene functional clustering showed that cellular biosynthetic processes, macromolecule biosynthetic and metabolic processes were negatively regulated. Biological processes such as the regulation of protein localization, protein transport and post-Golgi vesicle-mediated transport, which may be used by viruses for their own replication cycle, including capsid packaging and virus particles release, were clearly strengthened. In persistent infection, gene functional clustering showed that biological processes such as heart development, muscle organ development, muscle tissue development and striated muscle tissue development were all involved in response to the viral persistent infection. These clustered GO biological processes might be a critical mechanism used by host cells to survive in the situation of viral persistence.

\section{Gene expression validation by using real-time RT-PCR}

To confirm the results obtained from microarray analysis, we performed qRT-PCR for a selected group of 33 DEGs. 
Table 2. Seven DEGs were expressed in a similar trends in human and mouse arrays

\begin{tabular}{|c|c|c|c|c|}
\hline \multirow{2}{*}{ Gene symbol } & \multirow{2}{*}{ Gene description } & \multirow{2}{*}{ GO biological process description } & \multicolumn{2}{|c|}{ Fold changes } \\
\hline & & & Human & Mouse \\
\hline \multicolumn{5}{|c|}{ Acute infection } \\
\hline Apex2 & apurinic/apyrimidinic endonuclease 2 & DNA repair & 2.27 & 5.59 \\
\hline Mllt3 & $\begin{array}{l}\text { myeloid/lymphoid or mixed-lineage leukemia (trithorax } \\
\text { homolog, Drosophila); translocated to, } 3\end{array}$ & DNA-dependent regulation of transcription, & 0.39 & 0.27 \\
\hline Tle1 & $\begin{array}{l}\text { transducin-like enhancer of split } 1 \text { (E(sp1) homolog, } \\
\text { Drosophila) }\end{array}$ & signal transduction & 2.21 & 2.07 \\
\hline \multicolumn{5}{|c|}{$\begin{array}{rr}\text { Persistent infection } \\
\end{array}$} \\
\hline Cep135 & centrosomal protein $135 \mathrm{kDa}$ & - & 0.38 & 0.40 \\
\hline Sema4c & $\begin{array}{l}\text { sema domain, immunoglobulin domain (Ig), transmem- } \\
\text { brane domain (TM) and short cytoplasmic domain, } \\
\text { (semaphorin) } 4 \mathrm{C}\end{array}$ & multicellular organismal development & 0.24 & 0.28 \\
\hline Ttn & titin & carbohydrate metabolic process & 3.41 & 2.20 \\
\hline Usp11 & ubiquitin specific peptidase 11 & ubiquitin-dependent protein catabolic process & 0.50 & 0.32 \\
\hline
\end{tabular}

Table 3. Common up-regulated GO biological processes were identified by human and mouse microarrays. The common GO biological processes were determined by DAVID bioinformatics resources. For analysis, the parameter "count" threshold is 2 and the "EASE" is 0.1

\begin{tabular}{|c|c|c|}
\hline Biological process & Up-regulated DEGs in human microarray & Up-regulated DEGs in mouse microarray \\
\hline \multicolumn{3}{|c|}{\begin{tabular}{|c|} 
Acute infection \\
\end{tabular}} \\
\hline $\begin{array}{l}\text { Establishment of protein } \\
\text { localization }\end{array}$ & $\begin{array}{l}\text { SNX19, GNPTG, MCM3AP, TMCO6, SEC24A, ATG9A, } \\
\text { C16ORF70, IPO11, TIMM50, HLA-DMA, PEX7, SCFD1, } \\
\text { BACE2, NPM1, ARCN1, MGEA5, NCKIPSD, RAMP1, } \\
\text { COPE, HDAC6 }\end{array}$ & $\begin{array}{l}\text { RAB4B, ARF5, SNX3, VPS37D, ERC1, MON2, COG1, } \\
\text { SNX11 }\end{array}$ \\
\hline $\begin{array}{l}\text { Negative regulation of biosynthetic } \\
\text { process }\end{array}$ & $\begin{array}{l}\text { SBNO2, ZNF593, PDGFB, DEDD, TLE1, MBD3, MXD4, } \\
\text { RPS14, BACE2, MGEA5, COMMD7, HDAC8, DNAJB6 }\end{array}$ & $\begin{array}{l}\text { GMNN, TH1L, HES7, TLE1, NFIC, ADIPOQ, FOXP2, } \\
\text { CDT1 }\end{array}$ \\
\hline $\begin{array}{l}\text { Negative regulation of cellular } \\
\text { biosynthetic process }\end{array}$ & $\begin{array}{l}\text { SBNO2, ZNF593, PDGFB, DEDD, TLE1, MBD3, MXD4, } \\
\text { RPS14, BACE2, MGEA5, COMMD7, HDAC8, DNAJB6 }\end{array}$ & $\begin{array}{l}\text { GMNN, TH1L, HES7, TLE1, NFIC, ADIPOQ, FOXP2, } \\
\text { CDT1 }\end{array}$ \\
\hline $\begin{array}{l}\text { Negative regulation of macromol- } \\
\text { ecule biosynthetic process }\end{array}$ & $\begin{array}{l}\text { SBNO2, ZNF593, DEDD, BACE2, RPS14, MGEA5, TLE1, } \\
\text { MBD3, COMMD7, HDAC8, DNAJB6, MXD4 }\end{array}$ & GMNN, TH1L, HES7, TLE1, NFIC, FOXP2, CDT1 \\
\hline $\begin{array}{l}\text { Negative regulation of macromol- } \\
\text { ecule metabolic process }\end{array}$ & $\begin{array}{l}\text { CLN3, SBNO2, ZNF593, DEDD, TLE1, ANAPC10, } \\
\text { MBD3, MXD4, BACE2, RPS14, NPM1, MGEA5, COM- } \\
\text { MD7, HDAC8, RPS27A, BUB3, DNAJB6, HDAC6 }\end{array}$ & $\begin{array}{l}\text { HNF4A, GMNN, TH1L, HES7, TLE1, NFIC, FOXP2, } \\
\text { CDT1 }\end{array}$ \\
\hline $\begin{array}{l}\text { Post-Golgi vesicle-mediated } \\
\text { transport }\end{array}$ & SCFD1, GBF1, C16ORF70, MUTED & $\mathrm{CHIC} 2, \mathrm{MON} 2$ \\
\hline Protein transport & $\begin{array}{l}\text { SNX19, GNPTG, MCM3AP, TMCO6, SEC24A, ATG9A, } \\
\text { C16ORF70, IPO11, TIMM50, HLA-DMA, PEX7, SCFD1, } \\
\text { BACE2, NPM1, ARCN1, MGEA5, NCKIPSD, RAMP1, } \\
\text { COPE, HDAC6 }\end{array}$ & $\begin{array}{l}\text { RAB4B, ARF5, SNX3, VPS37D, ERC1, MON2, COG1, } \\
\text { SNX11 }\end{array}$ \\
\hline \multicolumn{3}{|c|}{ Persistent infection } \\
\hline Heart development & CHD7, ID1, PPP3CB, ID3, TTN, FOXP1 & MYO18B, NOTCH1, NODAL, TTN, POFUT1, GJA5 \\
\hline Muscle organ development & $\begin{array}{l}\text { IGSF8, CRYAB, FHL1, MET, TEAD4, PPP3CB, MYLPF, } \\
\text { HBEGF, TTN, CDK5, FOXP1 }\end{array}$ & LIF, MYO18B, MUSK, TTN, CHAT, FOXP2 \\
\hline Muscle tissue development & IGSF8, PPP3CB, MYLPF, TTN, CDK5, FOXP1 & MYO18B, MUSK, TTN, CHAT, FOXP2 \\
\hline One-carbon metabolic process & ATF7IP, FOS, GSPT1, AHCYL1, PCMT1 & SUV420H2, TRMT11, AHCYL2, HELLS \\
\hline Striated muscle tissue development & IGSF8, PPP3CB, MYLPF, TTN, CDK5, FOXP1 & MYO18B, MUSK, TTN, CHAT, FOXP2 \\
\hline
\end{tabular}

Three of them (Apex3, Ttn and Usp11) were selected from Table 2 (Co-DEGs). Fifteen genes were selected from Table S1 (Significant DEGs in human microarray) and the remaining ones were from Table S2 (Significant DEGs in mouse microarray). The data showed that all genes selected had similar expression patterns in both human and mouse microarrays (Fig. 4). Especially, the expression patterns of 7 genes (HPS1, DDX58 and KPTN in acute infection (Fig. 4a)); 
(a)

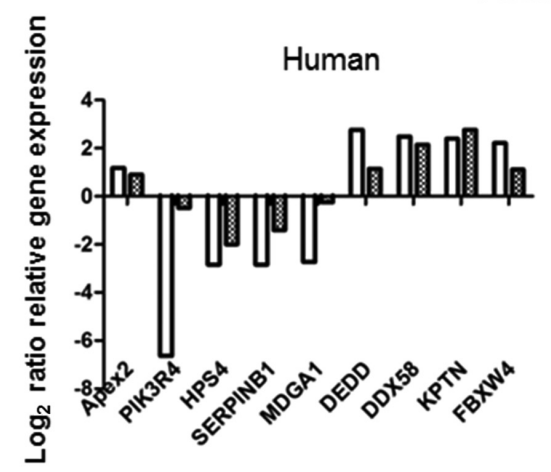

Acute infection

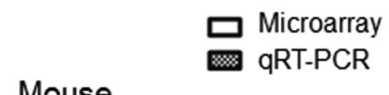

Mouse

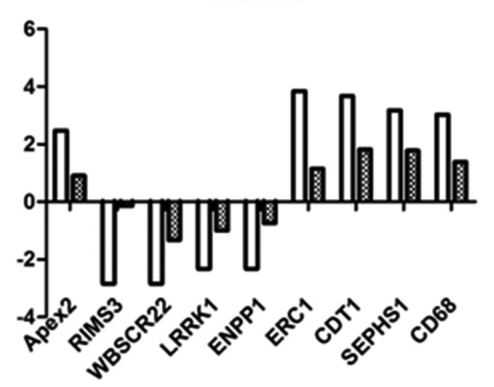

(b)

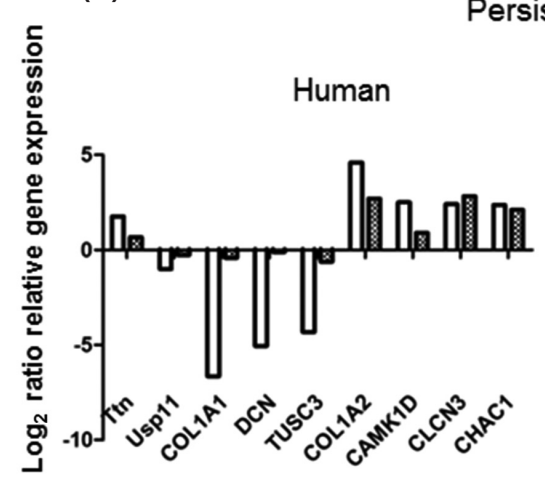

Persistent infection

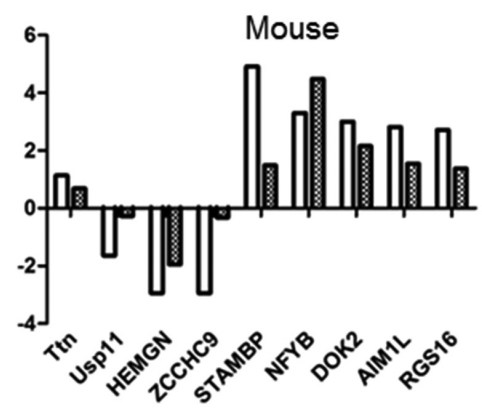

Fig. 4

Validation of gene expression in human and mouse microarrays

(a) Validation of gene expression in acute infection by using qRT-PCR. (b) Validation of gene expression in persistent infection by using qRT-PCR. Mockinfected sample was used a control for the FMDV infected samples. The transcript levels revealed by qRT-PCR were normalized against the housekeeping gene GAPDH. The $y$-axis represented $\log _{2}$ ratio of fold changes. Each reaction was repeated in duplicates.

CLCN3, CHAC1, NFYB, and DOK2 in persistent infection (Fig. 4b) were almost consistent with those in microarrays. Although the fold change of most of genes in qRT-PCR test was a little smaller than that in microarray, the expression patterns of these genes were quite similar.

\section{Discussion}

Due to the lack of native gene arrays for Syrian hamster, we used human and mouse microarrays to study the gene expression profiles in BHK-21 cells that were acutely and persistently infected with FMDV. On average, about $10 \%$ of the total probes in each array could be obviously detected in virus-infected or uninfected BHK-21 cell samples. Furthermore, expression changes of some genes in the FMDVinfected cells, compared with the uninfected BHK-21 cells, could be detected by using either human or mouse microarrays or the combination of the two types of microarrays. This suggested that the corresponding sequences of Syrian hamster at the specific locations are conserved with those from either human or mouse.

We used two-channel microarrays to hybridize the cDNAs from virus-infected and uninfected cells. The cDNAs of uninfected BHK-21 cell samples were labeled with Cy3 in red, while the cDNAs of virus-infected ones were labeled with Cy5 in green. Therefore, microarrays with probes hybridizing cDNAs of both virus-infected and uninfected samples would show yellow color. MA Plot not only showed the results of hybridization from two-channel microarrays, but also presented the fluorescence intensity of Cy3 and Cy5 channels. Hence, this could be an appropriate method to provide us an overall view of the cross-species hybridization results.

As we have shown, in both acute and persistent infections human microarrays hybridized many more (about 3 times) targets compared to mouse microarrays. In order to further investigate whether a transcriptome of Syrian hamster had higher homology to that of human, we cloned ten genes from BHK-21 cells, sequenced them and ran sequence alignment among Syrian hamster, human and mouse. The result 
showed that nine of the ten cloned genes had higher identity with mouse as compared to human corresponding genes (Table S3). These data demonstrated that Syrian hamster was more homologous to mouse than human, although human microarrays could hybridize more cDNAs of BHK-21 cells than mouse microarrays.

It has been recognized that genes with striking fold changes in microarray test are usually more valuable to investigators. We defined significant differentially expressed genes, of which the fold changes were larger than 4 or smaller than 0.25. Common and unique significant genes that might be related to the mechanisms underlying their own specific infection patterns were identified in both human and mouse microarrays. For example, in acute infection, ubiquitin-related genes, such as RNF139 and KPTN, and virus-responsive genes, such as DDX58, were identified in human microarray, while exocytosis genes, such as RIMS3, and immune genes such as CD68, were identified in mouse microarray. In persistent infection, multicellular organismal development genes, such as COL1A1, SERPINF1 and COL1A2, and calcium-dependent cell-cell adhesion genes, such as CDH11 were identified in human microarray. The most significant up-regulated gene STAMBP, which may participate in assisting persistently infected cells to survive, was identified in mouse microarray.

We then analyzed the common DEGs (co-DEGs) shared by human and mouse microarrays. Seven genes found to be differentially expressed in persistent infection had similar fold changes in human and mouse arrays, with 3 of them in acute infection and 4 in persistent infection. These coDEGs could be valuable in further investigation. To further confirm the results obtained with the microarray analysis, we performed qRT-PCR for a selected group of DEGs. We selected 33 genes, 3 (Apex2, Ttn and Usp11) of which were selected from Table 2 and the remaining were selected from Table S1 and Table S2. The results of qRT-PCR for acute and persistent infections presented great consistency in both human and mouse microarrays (Fig. 4, Table S5).

Finally, we used DAVID bioinformatics resources tool to analyze the common genes involved in biological processes between human and mouse microarrays. The gene functional clustering analysis of these two microarrays gave us better understanding of the unique biological processes taken by host cells in acute and persistent infections. Evidently, various biosynthetic and metabolic processes were negatively regulated in acute infection. Genes involved in multicellular organismal development processes were clustered in persistent infection. These results are valuable to explore the possible mechanisms taken by host BHK-21 cells in each infection pattern.

In summary, microarray studies in four BHK-21 cell samples (infected or uninfected) were performed with human and mouse oligonucleotide arrays. Surprisingly, in hybridization with the cDNAs from FMDV-infected BHK-
21 cells, human arrays are more informative than mouse arrays. Similar hybridization results were also reported in a previous study. The sequence alignments of ten genes cloned from BHK-21 cells confirmed that Syrian hamster is evolutionarily closer to mouse than human beings. Therefore, the combined use of human and mouse microarrays is more informative than the use of either array, which would be helpful for investigators to establish more complete gene expression profiles for other species.

Acknowledgement. The authors gratefully acknowledge KangChen Bio-tech (Shanghai, China) and China Infrastructure of Cell Line Resources for technical supports. This work was supported by the National Natural Sciences Foundation of China (No. 31370185) and the National Basic Research Program of China (No. NSTICR14, NSTI-CR15).

Supplementary information is available in the online version of the paper.

\section{References}

Adjaye J, Herwig R, Herrmann D, Wruck W, Benkahla A, Brink TC, Nowak M, Carnwath JW, Hultschig C, Niemann H, Lehrach H (2004): Cross-species hybridisation of human and bovine orthologous genes on high density cDNA microarrays. BMC Genomics 5, 83. https://doi. org/10.1186/1471-2164-5-83

Arzt J, Baxt B, Grubman MJ, Jackson T, Juleff N, Rhyan J, Rieder E, Waters R, Rodriguez LL (2011): The pathogenesis of foot-andmouth disease II: viral pathways in swine, small ruminants, and wildlife; myotropism, chronic syndromes, and molecular virus-host interactions. Transbound. Emerg. Dis. 58, 305-326. https://doi.org/10.1111/j.1865-1682.2011.01236.x

Balamurugan V, Kumar RM, Suryanarayana VV (2004): Past and present vaccine development strategies for the control of foot-and-mouth disease. Acta Virol. 48, 201-214.

Bar-Or C, Bar-Eyal M, Gal TZ, Kapulnik Y, Czosnek H, Koltai H (2006): Derivation of species-specific hybridization-like knowledge out of cross-species hybridization results. BMC Genomics 7, 110. https://doi.org/10.1186/1471-2164-7-110

Brodsky LI, Jacob-Hirsch J, Avivi A, Trakhtenbrot L, Zeligson S, Amariglio N, Paz A, Korol AB, Band M, Rechavi G, Nevo E (2005): Evolutionary regulation of the blind subterranean mole rat, Spalax, revealed by genome-wide gene expression. Proc. Natl. Acad. Sci. USA 102, 17047-17052. https://doi.org/10.1073/pnas.0505043102

Brown F (1999): Foot-and-mouth disease and beyond: vaccine design, past, present and future. Arch. Virol. (Suppl.) 15, 179-188. https://doi.org/10.1007/978-3-7091-6425-9_13

Cai KJ, Meng QL, Qiao J, Huang J, Zhang ZC, Wang GC, Wang JW, Chen CF (2013): Expression of bovine Mx1 protein inhibits the replication of foot-and-mouth disease virus in BHK-21 cells. Acta Virol. 57, 429-434. https://doi. org/10.4149/av $2013 \quad 04 \quad 429$ 
Chalmers AD, Goldstone K, Smith JC, Gilchrist M, Amaya E, Papalopulu N (2005): A Xenopus tropicalis oligonucleotide microarray works across species using RNA from Xenopus laevis. Mech. Dev. 122, 355-363. https://doi. org/10.1016/j.mod.2004.09.004

Clarke JB (1983): Transformation and foot and mouth disease virus (FMDV) productivity of some BHK cell lines. Acta Virol. 27, 534.

de la Torre JC, Davila M, Sobrino F, Ortin J, Domingo E (1985): Establishment of cell lines persistently infected with footand-mouth disease virus. Virology 145, 24-35. https:// doi.org/10.1016/0042-6822(85)90198-9

de la Torre JC, Martinez-Salas E, Diez J, Domingo E (1989): Extensive cell heterogeneity during persistent infection with foot-and-mouth disease virus. J. Virol. 63, 59-63.

de la Torre JC, Martinez-Salas E, Diez J, Villaverde A, Gebauer F, Rocha E, Davila M, Domingo E (1988): Coevolution of cells and viruses in a persistent infection of foot-and-mouth disease virus in cell culture. J. Virol. 62, 2050-2058.

Gilad Y, Rifkin SA, Bertone P, Gerstein M, White KP (2005): Multi-species microarrays reveal the effect of sequence divergence on gene expression profiles. Genome Res. 15, 674-680. https://doi.org/10.1101/gr.3335705

Grubman MJ, Baxt B (2004): Foot-and-mouth disease. Clin. Microbiol. Rev. 17, 465-493. https://doi.org/10.1128/ CMR.17.2.465-493.2004

Held M, Gase K, Baldwin IT (2004): Microarrays in ecological research: a case study of a cDNA microarray for plantherbivore interactions. BMC Ecol. 4, 13. https://doi. org/10.1186/1472-6785-4-13

Herrera M, Grande-Perez A, Perales C, Domingo E (2008): Persistence of foot-and-mouth disease virus in cell culture revisited: implications for contingency in evolution. J. Gen. Virol. 89, 232-244. https://doi.org/10.1099/vir.0.83312-0

Huang da W, Sherman BT, Lempicki RA (2009a): Bioinformatics enrichment tools: paths toward the comprehensive functional analysis of large gene lists. Nucleic Acids Res. 37, 1-13. https://doi.org/10.1093/nar/gkn923

Huang da W, Sherman BT, Lempicki RA (2009b): Systematic and integrative analysis of large gene lists using DAVID bioinformatics resources. Nat. Protoc. 4, 44-57.

Huang X, Li Y, Fang H, Zheng C (2011): Establishment of persistent infection with foot-and-mouth disease virus in BHK-21 cells. Virol. J. 8, 169. https://doi.org/10.1186/1743-422X-8-169

Ji W, Zhou W, Gregg K, Yu N, Davis S (2004): A method for cross-species gene expression analysis with high-density oligonucleotide arrays. Nucleic Acids Res. 32, e93. https:// doi.org/10.1093/nar/gnh084

Mahy BW (2005): Introduction and history of foot-and-mouth disease virus. Curr. Top. Microbiol. Immunol. 288, 1-8. https://doi.org/10.1007/3-540-27109-0 1

Martin Hernandez AM, Carrillo EC, Sevilla N, Domingo E (1994): Rapid cell variation can determine the establishment of a persistent viral infection. Proc. Natl. Acad. Sci. USA 91, 3705-3709. https://doi.org/10.1073/pnas.91.9.3705

Nagpal S, Karaman MW, Timmerman MM, Ho VV, Pike BL, Hacia JG (2004): Improving the sensitivity and specificity of gene expression analysis in highly related organisms through the use of electronic masks. Nucleic Acids Res. 32, e51. https://doi.org/10.1093/nar/gnh048

Neufeld JD, Mohn WW, de Lorenzo V (2006): Composition of microbial communities in hexachlorocyclohexane $(\mathrm{HCH})$ contaminated soils from Spain revealed with a habitatspecific microarray. Environ. Microbiol. 8, 126-140. https://doi.org/10.1111/j.1462-2920.2005.00875.x

Nieto-Diaz M, Pita-Thomas W, Nieto-Sampedro M (2007): Crossspecies analysis of gene expression in non-model mammals: reproducibility of hybridization on high density oligonucleotide microarrays. BMC Genomics 8, 89. https://doi.org/10.1186/1471-2164-8-89

Nowrousian M, Ringelberg C, Dunlap JC, Loros JJ, Kuck U (2005): Cross-species microarray hybridization to identify developmentally regulated genes in the filamentous fungus Sordaria macrospora. Mol. Genet. Genomics 273, 137-149. https://doi.org/10.1007/s00438-005-1118-9

Nuzhdin SV, Wayne ML, Harmon KL, McIntyre LM (2004): Common pattern of evolution of gene expression level and protein sequence in Drosophila. Mol. Biol. Evol. 21, 1308-1317. https://doi.org/10.1093/molbev/msh128

Renn SC, Aubin-Horth N, Hofmann HA (2004): Biologically meaningful expression profiling across species using heterologous hybridization to a cDNA microarray. BMC Genomics 5, 42. https://doi.org/10.1186/1471-2164-5-42

Rifkin SA, Kim J, White KP (2003): Evolution of gene expression in the Drosophila melanogaster subgroup. Nat. Genet. 33, 138-144. https://doi.org/10.1038/ng1086

Saetre P, Lindberg J, Leonard JA, Olsson K, Pettersson U, Ellegren H, Bergstrom TF, Vila C, Jazin E (2004): From wild wolf to domestic dog: gene expression changes in the brain. Brain Res. Mol. Brain Res. 126, 198-206. https://doi. org/10.1016/j.molbrainres.2004.05.003

Sen AK, Saha SN (1994): Development of an effective vaccine against foot-and-mouth disease with partially purified and concentrated virus antigen. Acta Virol. 38, 17-19.

Vallee M, Robert C, Methot S, Palin MF, Sirard MA (2006): Cross-species hybridizations on a multi-species cDNA microarray to identify evolutionarily conserved genes expressed in oocytes. BMC Genomics 7, 113. https://doi. org/10.1186/1471-2164-7-113

Walker SJ, Wang Y, Grant KA, Chan F, Hellmann GM (2006): Long versus short oligonucleotide microarrays for the study of gene expression in nonhuman primates. J. Neurosci. Methods 152, 179-189. https://doi.org/10.1016/j. jneumeth.2005.09.007

Wang F, Kuang Y, Salem N, Anderson PW, Lee Z (2009): Crossspecies hybridization of woodchuck hepatitis viral infection-induced woodchuck hepatocellular carcinoma using human, rat and mouse oligonucleotide microarrays. J. Gastroenterol. Hepatol. 24, 605-617. https://doi. org/10.1111/j.1440-1746.2008.05581.x

Zhang H, Li Y, Huang X, Zheng C (2013): Global transcriptional analysis of model of persistent FMDV infection reveals critical role of host cells in persistence. Vet. Microbiol. 162, 321-329. https://doi.org/10.1016/j.vetmic.2012.09.007 


\title{
Supplementary information
}

\section{Cross-species hybridization of foot-and-mouth disease virus-infected BHK-21 cells using human and mouse oligonucleotide microarrays}

\author{
H. ZHANG ${ }^{1,2}$, Q. WANG ${ }^{3}$, L. HAN ${ }^{1}$, C. ZHENG ${ }^{1}$, CH. SHEN ${ }^{1}$
}

${ }^{1}$ State Key Laboratory of Virology, College of Life Sciences, Wuhan University, Wuhan, Hubei 430072, P. R. China; ${ }^{2}$ Bioengineering Department, Wuhan Bioengineering Institute, Luoyang Economy Development Zone, Wuhan, Hubei 430415, P. R. China; ${ }^{3} \mathrm{Center}$ for Computational and Integrative Biology, Massachusetts General Hospital, Harvard Medical School, Boston, MA 02114, USA

Received August 8, 2016; revised December 19, 2016; accepted June 27, 2017

Table S1. Significant DEGs in human microarray

\begin{tabular}{|c|c|c|c|c|}
\hline Fold change & Gene description & Gene symbol & $\begin{array}{l}\text { GO biological process } \\
\text { description }\end{array}$ & UniGene ID \\
\hline \multicolumn{5}{|c|}{ Acute infection } \\
\hline 0.01 & phosphoinositide-3-kinase, regulatory subunit 4 & PIK3R4 & $\begin{array}{l}\text { protein amino acid phosphoryla- } \\
\text { tion }\end{array}$ & Hs. 149032 \\
\hline 0.14 & Hermansky-Pudlak syndrome 4 & HPS4 & protein targeting & Hs. 474436 \\
\hline 0.14 & Serpin B1 & SERPINB1 & - & - \\
\hline 0.15 & $\begin{array}{l}\text { MAM domain containing glycosylphosphatidyli- } \\
\text { nositol anchor } 1\end{array}$ & MDGA1 & - & Hs. 437993 \\
\hline 0.15 & chromosome 12 open reading frame 36 & C12orf36 & - & Hs. 448717 \\
\hline 0.18 & solute carrier family 2 , member 8 & SLC2A8 & carbohydrate metabolic process & Hs.179522 \\
\hline 0.18 & solute carrier family 38 , member 4 & SLC38A4 & ion transport & Hs. 446077 \\
\hline 0.18 & ring finger protein 139 & RNF139 & ubiquitin cycle & Hs.632057 \\
\hline 0.19 & SET binding protein 1 & SETBP1 & - & Hs. 435458 \\
\hline 4.17 & transmembrane 6 superfamily member 1 & TM6SF1 & - & Hs.513094 \\
\hline 4.44 & ribosomal protein $\mathrm{L} 37$ & RPL37 & translation & Hs. 80545 \\
\hline 4.63 & F-box and WD repeat domain containing 4 & FBXW4 & ubiquitin cycle & Hs. 500822 \\
\hline 4.66 & coiled-coil domain containing 106 & CCDC106 & - & Hs. 82482 \\
\hline 4.89 & transmembrane and coiled-coil domains 6 & TMCO6 & - & Hs. 651145 \\
\hline 5.28 & kaptin & KPTN & cell motility & Hs. 25441 \\
\hline 5.62 & DEAD (Asp-Glu-Ala-Asp) box polypeptide 58 & DDX58 & response to virus & Hs. 190622 \\
\hline 5.90 & zinc finger, DHHC-type containing 5 & ZDHHC5 & - & Hs.27239 \\
\hline 6.77 & death effector domain containing & DEDD & transcription & Hs. 517342 \\
\hline \multicolumn{5}{|c|}{ Persistent infection } \\
\hline 0.002 & cadherin 11 & CDH11 & cell adhesion & Hs.116471 \\
\hline 0.01 & collagen, type I, alpha 1 & COL1A1 & skeletal system development & Hs. 172928 \\
\hline 0.03 & defective in cullin neddylation 1 & $\mathrm{DCN}$ & carbohydrate metabolic process & Hs. 503716 \\
\hline 0.03 & metallothionein $1 \mathrm{E}$ & MT1E & negative regulation of growth & Hs. 534330 \\
\hline 0.04 & islet cell autoantigen 1 & ICA1 & neurotransmitter transport & Hs. 487561 \\
\hline 0.05 & oxysterol binding protein-like 6 & OSBPL6 & lipid transport & Hs. 318775 \\
\hline 0.05 & tumor suppressor candidate 3 & TUSC3 & $\begin{array}{l}\text { post-translational protein modi- } \\
\text { fication }\end{array}$ & Hs. 426324 \\
\hline 0.05 & SAC3 domain containing 1 & SAC3D1 & $\begin{array}{l}\text { negative regulation of JAK-STAT } \\
\text { cascade }\end{array}$ & Hs.23642 \\
\hline
\end{tabular}


Table S1 (continued)

\begin{tabular}{|c|c|c|c|c|}
\hline Fold change & Gene description & Gene symbol & $\begin{array}{l}\text { GO biological process } \\
\text { description }\end{array}$ & UniGene ID \\
\hline 0.05 & serpin peptidase inhibitor, clade F & SERPINF1 & $\begin{array}{l}\text { multicellular organismal develop- } \\
\text { ment }\end{array}$ & Hs. 532768 \\
\hline 5.15 & ChaC, cation transport regulator homolog 1 & CHAC1 & $\begin{array}{l}\text { negative regulation of Notch signal- } \\
\text { ing pathway }\end{array}$ & Hs. 155569 \\
\hline 5.37 & chloride channel 3 & CLCN3 & ion transmembrane transport & Hs. 481186 \\
\hline 5.62 & solute carrier family 24, member 3 & SLC24A3 & transmembrane transport & Hs. 654790 \\
\hline 5.64 & upstream transcription factor 2 & FOS & $\begin{array}{l}\text { toll-like receptor signaling path- } \\
\text { way }\end{array}$ & Hs. 454534 \\
\hline 5.67 & plasma glutamate carboxypeptidase & PGCP & proteolysis & Hs. 156178 \\
\hline 5.69 & $\begin{array}{l}\text { calcium/calmodulin-dependent protein kinase } \\
\text { ID }\end{array}$ & CAMK1D & protein phosphorylation & Hs.659517 \\
\hline 6.56 & Ras-related associated with diabetes & RRAD & GTP catabolic process & Hs.1027 \\
\hline 6.80 & adenosylhomocysteinase-like 1 & AHCYL1 & mRNA polyadenylation & Hs. 485365 \\
\hline 24.45 & collagen, type I, alpha 2 & COL1A2 & skeletal system development & Hs. 489142 \\
\hline
\end{tabular}

Table S2. Significant DEGs in mouse microarray

\begin{tabular}{|c|c|c|c|c|}
\hline Fold change & Gene description & Gene symbol & $\begin{array}{l}\text { GO biological process } \\
\text { description }\end{array}$ & UniGene ID \\
\hline \multicolumn{5}{|c|}{ Acute infection } \\
\hline 0.12 & adult male cecum cDNA, unclassifiable & 9130221J18RIK & - & Mm.207250 \\
\hline 0.14 & regulating synaptic membrane exocytosis 3 & RIMS3 & exocytosis & Mm.479706 \\
\hline 0.14 & $\begin{array}{l}\text { Williams Beuren syndrome chromosome region } \\
22\end{array}$ & WBSCR22 & rRNA processing & Mm.439878 \\
\hline 0.20 & leucine-rich repeat kinase 1 & LRRK1 & protein phosphorylation & Mm.33284 \\
\hline 0.20 & $\begin{array}{l}\text { ectonucleotide pyrophosphatase/phosphodi- } \\
\text { esterase } 1\end{array}$ & ENPP1 & $\begin{array}{l}\text { generation of precursor metabo- } \\
\text { lites and energy }\end{array}$ & Mm.485841 \\
\hline 0.24 & hypothetical LOC432488 & LOC432488 & - & Mm.32833 \\
\hline 0.24 & 7 days embryo whole body cDNA, unclassifiable & C430019N01RIK & - & Mm.447782 \\
\hline 0.24 & $\begin{array}{l}\text { TGF-beta activated kinase } 1 / \text { MAP } 3 \mathrm{~K} 7 \text { binding } \\
\text { protein } 2\end{array}$ & TAB2 & $\begin{array}{l}\text { MyD88-dependent toll-like recep- } \\
\text { tor signaling pathway }\end{array}$ & \\
\hline 0.25 & absent in melanoma 1-like & AIM1L & - & Mm.240080 \\
\hline 8.17 & CD68 antigen & CD68 & $\begin{array}{l}\text { cellular response to organic sub- } \\
\text { stance }\end{array}$ & Mm.15819 \\
\hline 9.07 & selenophosphate synthetase 1 & SEPHS1 & $\begin{array}{l}\text { cellular protein modification proc- } \\
\text { ess }\end{array}$ & Mm.34329 \\
\hline 10.37 & T-cell leukemia/lymphoma 1B, 1 & TCL1B1 & - & Mm.241933 \\
\hline 11.43 & adult male testis cDNA, unclassifiable & 1700018G05RIK & - & Mm.138009 \\
\hline 11.67 & ADAM Metallopeptidase Domain 28 & ADAM28 & proteolysis & Mm.117450 \\
\hline 12.81 & $\begin{array}{l}\text { chromatin licensing and DNA replication fac- } \\
\text { tor } 1\end{array}$ & CDT1 & DNA replication checkpoint & Mm.21873 \\
\hline 13.73 & retinol binding protein 7 , cellular & RBP7 & - & Mm.46023 \\
\hline 14.42 & ELKS/RAB6-interacting/CAST family member 1 & ERC1 & $\begin{array}{l}\text { regulation of transcription, DNA- } \\
\text { templated }\end{array}$ & Mm.288860 \\
\hline 16.46 & ADAMTS-like 1 & ADAMTSL1 & - & Mm.331690 \\
\hline \multicolumn{5}{|c|}{ Persistent infection } \\
\hline 0.10 & cAMP responsive element binding protein 5 & CREB5 & $\begin{array}{l}\text { positive regulation of transcription, } \\
\text { DNA-templated }\end{array}$ & Mm.321138 \\
\hline 0.11 & mus musculus expressed sequence AW555464 & AW555464 & - & Mm.23689 \\
\hline 0.11 & intersectin 1 (SH3 domain protein) & ITSN1 & $\begin{array}{l}\text { positive regulation of apoptotic } \\
\text { process }\end{array}$ & Mm.40546 \\
\hline 0.12 & transmembrane protein 62 & TMEM62 & - & Mm.315212 \\
\hline
\end{tabular}


Table S2 (continued)

\begin{tabular}{|c|c|c|c|c|}
\hline Fold change & Gene description & Gene symbol & $\begin{array}{l}\text { GO biological process } \\
\text { description }\end{array}$ & UniGene ID \\
\hline 0.13 & Eph receptor $\mathrm{B} 3$ & EPHB3 & $\begin{array}{l}\text { transmembrane receptor protein } \\
\text { tyrosine kinase signaling pathway }\end{array}$ & Mm.6972 \\
\hline 0.13 & hemogen & HEMGN & $\begin{array}{l}\text { multicellular organismal develop- } \\
\text { men }\end{array}$ & Mm.25793 \\
\hline 0.13 & eyes absent 1 homolog & EYA1 & $\begin{array}{l}\text { establishment of mitotic spindle } \\
\text { orientation }\end{array}$ & Mm.250185 \\
\hline 0.13 & N-sulfoglucosamine sulfohydrolase & SGSH & $\begin{array}{l}\text { glycosaminoglycan catabolic proc- } \\
\text { ess }\end{array}$ & Mm.103788 \\
\hline 0.13 & zinc finger, CCHC domain containing 9 & ZCCHC9 & $\begin{array}{l}\text { negative regulation of phosphatase } \\
\text { activity }\end{array}$ & Mm.52356 \\
\hline 6.45 & mus musculus RIKEN cDNA E130309D02 gene & E130309D02RIK & - & Mm.440105 \\
\hline 6.54 & regulator of G-protein signaling 16 & RGS16 & $\begin{array}{l}\text { positive regulation of GTPase } \\
\text { activity }\end{array}$ & Mm.181709 \\
\hline 6.69 & junction plakoglobin & JUP & $\begin{array}{l}\text { negative regulation of transcrip- } \\
\text { tion from RNA polymerase II } \\
\text { promoter }\end{array}$ & Mm.299774 \\
\hline 6.95 & $\begin{array}{l}\text { sugano mouse kidney mkia Mus musculus cDNA } \\
\text { clone }\end{array}$ & AI987986 & - & Mm.443881 \\
\hline 7.01 & absent in melanoma 1-like & AIM1L & - & Mm.240080 \\
\hline 7.65 & $\begin{array}{l}\text { gamma-aminobutyric acid (GABA) A receptor, } \\
\text { pi }\end{array}$ & GABRP & transport & Mm.99989 \\
\hline 8.03 & docking protein 2 & DOK2 & signal transduction & Mm.243323 \\
\hline 9.85 & nuclear transcription factor-Y beta & NFYB & transcription, DNA-templated & Mm.245998 \\
\hline 30.16 & STAM binding protein & STAMBP & mitotic cytokinesis & Mm.32801 \\
\hline
\end{tabular}

Table S3. Homology comparation of Syrian hamster genes to human and mouse by sequence alignment

\begin{tabular}{|c|c|c|c|c|c|c|c|}
\hline & $\begin{array}{c}\text { Hamster cloned } \\
\text { gene }\end{array}$ & $\begin{array}{l}\text { Cloned sequence } \\
\text { lenth (bp) }\end{array}$ & Species & $\begin{array}{c}\text { Genebank } \\
\text { number }\end{array}$ & Gene lenth (bp) & $\begin{array}{c}\text { Sequence identity } \\
(\%)\end{array}$ & $\begin{array}{c}\text { More close to } \\
\text { hamster }\end{array}$ \\
\hline \multirow{2}{*}{1} & \multirow{2}{*}{ EBP } & \multirow{2}{*}{693} & Human & NM_006579 & 1191 & 80.09 & \multirow{2}{*}{ Mouse } \\
\hline & & & Mouse & NM_007898 & 1755 & 87.30 & \\
\hline \multirow{2}{*}{2} & \multirow{2}{*}{ USP11 } & \multirow{2}{*}{1661} & Human & NM_004651 & 3300 & 84.23 & \multirow{2}{*}{ Mouse } \\
\hline & & & Mouse & NM_145628 & 3464 & 92.66 & \\
\hline \multirow{2}{*}{3} & \multirow{2}{*}{ GNG12 } & \multirow{2}{*}{219} & Human & NM_018841 & 4427 & 86.30 & \multirow{2}{*}{ Mouse } \\
\hline & & & Mouse & NM_001177560 & 4264 & 94.98 & \\
\hline \multirow{2}{*}{4} & \multirow{2}{*}{ FKBP1A } & \multirow{2}{*}{327} & Human & NM_000801 & 1643 & 94.80 & \multirow{2}{*}{ Mouse } \\
\hline & & & Mouse & NM_008019 & 1657 & 96.02 & \\
\hline \multirow{2}{*}{5} & \multirow{2}{*}{ TUBB2A } & \multirow{2}{*}{1317} & Human & NM_001069 & 1621 & 90.66 & \multirow{2}{*}{ Mouse } \\
\hline & & & Mouse & NM_009450 & 1638 & 92.94 & \\
\hline \multirow{2}{*}{6} & \multirow{2}{*}{ TUBA1B } & \multirow{2}{*}{1349} & Human & NM_006082 & 1771 & 94.96 & \multirow{2}{*}{ Mouse } \\
\hline & & & Mouse & NM_011654 & 1747 & 96.44 & \\
\hline \multirow{2}{*}{7} & \multirow{2}{*}{ APEX2 } & \multirow{2}{*}{98} & Human & NM_014481 & 2095 & 92.86 & \multirow{2}{*}{ Human } \\
\hline & & & Mouse & NM_029943 & 1903 & 91.84 & \\
\hline \multirow{2}{*}{8} & \multirow{2}{*}{ LEPREL4 } & \multirow{2}{*}{69} & Human & NM_006455 & 2619 & 91.30 & \multirow{2}{*}{ Mouse } \\
\hline & & & Mouse & NM_176830 & 1903 & 92.75 & \\
\hline \multirow{2}{*}{9} & \multirow{2}{*}{ RPL34 } & \multirow{2}{*}{118} & Human & NM_000995 & 918 & 94.92 & Моuse \\
\hline & & & Mouse & NM_026724 & 628 & 96.61 & iviouse \\
\hline & & & Human & NM_001025 & 3325 & 89.42 & \\
\hline 10 & RPSL3 & 189 & Mouse & NM_024175 & 572 & 94.18 & Mouse \\
\hline
\end{tabular}


EBP1:

ATGACCACCAATTCGGGCCCGGTACACCCAT ACTGGCCCAGGCACCTGAAGCTGGACAACTTT GTGCCTAATGACCTCCCTACCTGGAATATCCT GGTTGGCCTATTCTCCATCTCTGGATTCCTAATTG TGGTCATGTGGCTGTTGTCCAGTCGAGTTTCCGT TGCCCCACTTGGAATTTGGCGTCGACTGGCCCTGT GCTGGTTTGCTGTGTGTGCGTTCATTCACCTTGT GATCGAGGGTTGGTTCTCTGTATACCATGACATC CTTCTTGAAGACCAAGCCGTCTTATCCCAACTCT GGA A A GA G TAT T C CA A GGGA GACA GCCGA TATATACTTAATGACGGCTTCATCGTCTCTAT GGAGACTGTCACAGCTGT TCTCTGGGGAC CACTCAGCCTGTGGGTAGTGATTGCCTTTCTC CGCCAACAACCTTACCGCTTTATCCTACAGCTCGT GATCTCTGTGGGCCAGATATACGGGGATGTGCTG TACTTCCTGACTGAGCTTCGTGATGGATTCCAGCAT GGGGAGCTCGGCCACCCCCTTTATTTCTGGTTT TACTTTGTTATCTTGAATGGCATATGGCTGGTGG TACCTGCAATCCTCGTGCTTGATGCTATAAAG CATCTCGCTCACGCCCAGAGCATGTTGGACAG CAAGGTTATGAAAACTAAGAGCAAACATAACTAA

USP11:

ATGGCGGCGGTCGCAGCGGACCCAGCTGCAGC TGCCATCCCGGCCTCGGCCGAGGAGGAAGAG AGACAGCACGAGGCAATGCCAGACCTGGACGA G C A G T G G C G C C A A G T C G A G A A T G G G C G AGAGCGTCCACTGCGAGCTGGCGAAAGCTGG T TCCT TGTGGAGCAACACTGGT T TAAACAG TGGGAGGTGTACGTGAAGGGAGGGGACCAGGA TGCTAACACCTTTCCTGGCTGTATCAACAATGC TGGGCTCTTCGAAGATCAGATAAACTGGCACC TCAGGGAGGGACTGCTAGAAGGAAATGATTATG TGCTGCTCCCAGCGACTTCATGGAATTACTTGG TCAGCTGGTATGGCCTAGAAGATGGCCAGCCA CCTATTGAGCGCAAGGTCATAGAACTTTCTGG TGTCCCAAAGGTGGAATTATACCCAATAGAGCTGC TGCTTGTCCAGCACAGTGATATGGAAACAGCTC TCACCAT TCAGT TCAGCCACACCGAT TCTC TGGACGTAGTCTTGCAAACAGCTCGGGAGCAGT TTCTGGTGGAGCCTCAGGAAGACACACGCCTC TGGATCAAGAACTCCGAGGGCTCCTTGGATCGAT TGTGTAACACACAAATCACACTGCTCGATGCCT CCCTTGAGACTGGACAGTTGGTCATCATGGAAA CCCGAAACAAAGATGGCACTTGGCCCAGCGC TCAGCTGTGTGGCATGGACAACATGCCAGAAGA GGATGAAGACTTCCAGGGCCAGCCGGGCATC TGTGGCCT TACCAATCTGGGCAACACGTGC TTCATGAACTCGGCCCTACAGTGCCTCAGCAA TGTGCCACAGCTCACGGAGTACTTTCTCAACAA CCGCTACCTGGAGGAGCTCAACTTCTGGAACCCT TTAGGCATGAAGGGTGAGCTTGCTGAGGCCTA
TGCAGATCTGGTAAAGCAGACCTGGTCTGGCTA C C A C C G C T C C A T T G T G C C GA A T G T G T T C A AGAACAAGGTTGGCCATTTTGCATCCCAGTTTC TGGGGTACCAGCAACATGACTCACAGGAACTGT TGTCATTCCTTCTGGATGGGCTACATGAGGACC TCAATCGTGTCAAAAAGAAAGAATATGTTGAGC TGTGCGATGGTGCTGGGCGCCCGGATTTGGAAG TGGCTCAGGAAGCCTGGCAAAACCACAAACGA CGAAATGATTCTGTGATTGTGGATACTTTCCA TGGCCTCTTCAAATCTACTCTGGTGTGCCCTGAT TGTGGCAATGTATCTGTGACCTTTGACCCCTTC TGCTACCTCAGCGTCCCACTGCCTGTCTGCT CCAGGAGGGTCTTGGAGGTCTTCTTTGTCCCCA TGGATCCCCGCCGCAAGCCAGAACAGCACCGGGT TGTGGTCCCCAAGAAAGGCAATATTTCAGATCTG TGTGTGGCTCTGTCCACACACACAAGTGTTGCA CCAGACAAGATGATAGTAGCTGATGTCTTCAGTCAC CGATTCTATAAGCTCTACCAGCTGGAGGATCCTCT GAGTAGCATCTTGGATCGGGATGATATCTTTGTATAT GAGGTGACTGGTCGGATTGAGCCTGTTGAGGGT TCAAGAGATGATATCGTGGTTCCTGTTTACCTGCGA GAGCGCACCCCATCCCGAGACTTCGACAACTC

GNG12:

ATGTCCAGCAAGACGGCAAGCACCAACAACA TAGCCCAGGCCAGGAGAACGGTGCAGCAGTTG AGACTGGAAGCCTCCATCGAAAGAATAAAGGTCT CCAAAGCATCTGCAGACCTGATGTCATACTGTGA GGAGCATGCCCGGA GCGACCCTCTACTGA TGGGCATACCGACCTCGGAAAACCCATTCAAGG ATAAGAAGACCTGCATCATCTTATAG

\section{FKBP1A:}

ATGGGAGTGCAGGTGGAAACCATCTCTCCTGG AGACGGACGCACCTTCCCGAAGCGCGGCCAGA CCTGCGTGGTGCACTACACGGGAATGCTTGAA GATGGAAAGAAATTTGATTCTTCTCGGGACA GAAACAAGCCTTTTAAGTTTATGCTAGGCAAG CAGGAGGTGATCCGAGGCTGGGAAGAAGGGG TAGCCCAGATGAGTGTGGGTCAGAGAGCCAAACT GACAATCTCCCCAGATTATGCCTATGGAGCCACT GGGCACCCAGGCATCATCCCACCACATGCCACTCT GGTTTTTGATGTGGAGCTTCTAAAACTGGAATGA

\section{TUBB2A(beta-tubulin)}

ATGCGTGAGATCGTGCACATCCAGGCGGGCCAG TGCGGCAACCAGATCGGCGCTAAGTTTTGGGA GGTGATAAGCGATGAGCATGGCATCGACCCCA CCGGCAGTTACCATGGTGACAGTGATTTGCAGC TGGAGAGAATCAATGTGTACTACAATGAAGC TGCTGGCAACAAATATGTACCTCGGGCCAT CCTGGTGGACCTGGAGCCTGGCACCATGGAC TCAGTGAGGTCAGGACCCTTCGGCCAGATC 
TTCAGGCCAGACAACTTTGTTTTCGGCCAGAGCG GTGCAGGAAACAACTGGGCAAGGGCCACTACACA GAGGGCGCTGAGCTGGTGGACTCCGTCCTGGATG TAGTGAGGAAGGAGTCTGAAAGCTGTGACTGTCTC CAGGGCTTCCAGCTGACCCACTCACTGGGGGGAG GCACTGGCTCAGGCATGGGGACCCTGCTCAT CAGCAAGATCCGAGAGGAGTACCCAGACCGCAT CATGAACACCTTCAGTGTCATGCCCTCACCCAAG GTCTCTGACACTGTGGTGGAGCCCTATAATGCCAC CCTCTCCGTGCACCAGCTGGTGGAGAACACAGAT GAAACCTATTCCATTGACAACGAGGCCCTGTACGA CATCTGCTTCCGCACCCTCAAGCTGACCACACCCA CATATGGCGACCTCAACCACCTGGTGTCAGCCAC CATGAGTGGGGTGACCACCTGCCTGCGCTTC CCAGGCCAGCTGAACGCAGACCTGCGCAAGCT GGCCGTGAACATGGTGCCCTTCCCACGCCTGCACT TCTTCATGCCAGGCTTCGCGCCTCTGACCAGCAG GGGCAGCCAGCAGTACCGAGCCCTGACAGTGCCT GAGCTCACCCAGCAGATGTTCGATGCCAAGAACAT GATGGCCGCCTGTGATCCCCGCCATGGGCGCTACTT GACAGTGGCTGCTGTGTTCCGGGGCCGCATGTC TATGAAGGAGGTGGACGAACAGATGCTTAATGTC CAAAACAAGAACAGCAGCTACTTTGTTGAGTGGAT TCCCAACAATGTGAAAACAGCTGTCTGTGACAT TCCACCTCGGGGTCTAAAAATGTCTGCCACCT TCATCGGCAACAGCACCGCCATTCAGGAGCTGT TCAAACGCATCTCTGAGCAGTTCACAGCCATGT TCCGACGCAAGGCTTTCCTGCACTGGTACACG GGTGAGGGCATGGATGAGATGGAGTTCACTGA GGCTGAGAGCAACATGAATGACCTGGTGTCTGAG TACCAGCAGTACCAGGATGCCACGGCTGAGGAG GAGGGCGAGGACGAGGCGTAGG

TUBA1B(alpha-tubulin)

ATGCGTGAGTGCATCTCCATCCACGTTGGCCA G G C T G G T G T C C A G A T C G G C A A T G C C T G C TGGGAGCTCTACTGCCTGGAACATGGCAT CCAGCCTGACGGCCAGATGCCAAGTGACAAGA CCATTGGGGGAGGAGATGACTCCT TCAACA CCTTCTTCAGTGAGACAGGCGCTGGCAAGCA TGTGCCCCGGGCTGTGTTCGTAGACCTGGAA CCCACAGTTATCGATGAAGTTCGCACTGGTACCTA CCGCCAGCTCTTCCACCCTGAGCAGCTCATCACA GGCAAGGAAGATGCTGCCAACAACTATGCCCG TGGCCACTACACCAT TGGCAAGGAGATCAT TGATCTTGTCTTGGACAGAATTCGCAAACTGGC TGACCAGTGCACGGGTCTTCAGGGCTTCCTGGTT TTCCACAGCTTTGGTGGGGGAACTGGCTCTGGG TTCACCTCCCTGCTGATGGAGCGGCTCTCTGT CGATTATGGAAAGAAGTCCAAGCTGGAGTTCT CCATCTACCCAGCCCCCCAGGTTTCCACCGC TGTGGTTGAGCCCTACAACTCCATCCTCACCA CCCACACCACCCTGGAGCACTCTGATTGTGCC
TTCATGGTAGACAATGAGGCCATCTATGACATC TGTCGTAGAAACCTCGACATTGAGCGCCCCA CCTACACTAACCTTAACCGCCTCATTAGCCAGAT TGTGTCTTCCATCACTGCTTCCCTCAGATTTGA TGGGGCCCTGAATGTTGACCTGACAGAGTTCC AGACCAACCTGGTACCCTACCCTCGCATCCAT TTCCCTCTGGCCACATATGCCCCTGTCATCTCTGC TGAGAAAGCCTACCATGAGCAGCTTTCTGTAGC AGAGATCACCAATGCCTGCTTTGAGCCAGCCAA CCAGATGGTGAAATGTGACCCTCGCCATGGTAAA TACATGGCTTGCTGCCTGCTGTACCGTGGGGATG TGGTTCCCAAAGATGTCAATGCTGCCATTGCCA CCATCAAGACCAAGCGCAGCATCCAGT T TG TGGACTGGTGCCCCACTGGCTTCAAGGTTGGCA TTAACTACCAGCCTCCCACCGTGGTACCTGG TGGAGACCTGGCCAAGGTCCAGAGAGCTGTG TGCATGCTGAGCAATACCACAGCCATCGCTGA GGCCTGGGCTCGCCTAGATCACAAGTTTGATC TGATGTATGCCAAGCGTGCCTTTGTGCACTGGTA TGTGGGTGAGGGCATGGAGGAAGGGGAGTTCTC TGAGGCCCGTGAAGACATGGCTGCCCTAGAGAA GGATTATGAGGAGGTTGGTGTGGATTCTGTTGA GGGAGAGGGTGAGGAAGAAGGAGAAGA

APEX2

ATTCGTCTGTCTCCAGGAAACCAAAGTGACCAG AGATGCTCTGACGGAGCCCCTGGCTATTGTTGA GGGCTATAACTCCTATTTCAGCTTCAGCCGCA

EBP

ACTGTCCCTGTGCTGGTTTGCTGTGTGTGCG TTCATTCACCTTGTGATCGAGGGTTGGTTCTCTG TGTACCATGACATCCTTCTTGAAGACTAAGCCGTC TTATCCCAACTCTGGAAAGAGTATTCCAAGGG AGACAGCCGATACATC

LEPREL4

GGTGGCTACTTCGTGGACAAGTTTGTGGCCACCA TGTATCACTACCTGCAGTTTGCCTACTACAAATGA

RPL34

CAGCGTTTGACATACCGACGTAGGCTTTCCT ACAACACAGCCTCTAACAAAACTAGGCTGTCT CGAACCCCTGGCAACAGGATCGTTTACCTTTATA CCAAGAAGGTTGGGAAAGCAC

RPS23

GATGGGCAAGTGTCGCGGTCTCCGTACTGCCC GGAAGCTCCGCAGTCACCGACGGGACCAGAAG TGGCATGATAAGCAGTACAAGAAAGCCCACC TGGGCACAGCCCTGAAGGCCAACCCCTTCGGAGG TGCCTCTCATGCGAAGGGAATTGTGCTGGAAAAA GTAGGGGTTGAAGCCAAACAGCCAAA 
Table S4. Primers for real-time RT-PCR validation

\begin{tabular}{|c|c|c|c|}
\hline Gene & Forward primer & Reverse primer & Product lenth $(b p)$ \\
\hline \multicolumn{4}{|c|}{ Acute infection } \\
\hline Apex2 & CGTCTGTCTCCAGGAAACCA & GCGGCTGAAGCTGAAATAGG & 94 \\
\hline \multicolumn{4}{|c|}{ Human microarray } \\
\hline PIK3R4 & AAAATAAGGTTCTGGGACT & GTAGGACACGGCTGGA & 81 \\
\hline HPS4 & GTGCGTGAACTGGAAACTC & GGTGGCTTCGGATAGGT & 91 \\
\hline SERPINB1 & TGCCAGGACGACACTC & TGGTGAAGGCTGCTGTA & 110 \\
\hline MDGA1 & GTGGCATCCCAGATAAGGC & CCAGAGTTTCGTTCACAGACAG & 87 \\
\hline DEDD & GTTTCCTGTTGCCACTTAC & CСTTACAAAGGCTATGCTC & 102 \\
\hline DDX58 & CTGGTGGCTTGTTGCT & GATTCTGCCGCTGTTC & 130 \\
\hline KPTN & GGTGGTGGGCATCACAT & CCAGGCTCATAGTCACAGTAAA & 81 \\
\hline FBXW4 & TCCTCCTACTATGGTGTTGTG & AGATGCCTGGTGGTGAAT & 125 \\
\hline \multicolumn{4}{|c|}{ Mouse microarray } \\
\hline RIMS3 & AGCAGCATTTCTGTCGTG & AGAGGCGGCGTATTCA & 134 \\
\hline WBSCR22 & GAAGAAGAGCAGGGAATGG & CCGTGTACTGGGTGTCAGG & 86 \\
\hline LRRK1 & AGGGCACAGAAGACAAACA & TCCCAAACCGCCAGTA & 81 \\
\hline ENPP1 & TCCTCGCTCCACCAA & ACATAGGCACGATATTAGA & 81 \\
\hline ERC1 & ATGAGTGACCGAATACAGC & TCAAGATTTCCAAGAGGC & 100 \\
\hline CDT1 & GGCTTTCCTTGCTTCTTT & AGGCACCTCGTCCACAT & 91 \\
\hline SEPHS1 & TAAACTCGCCCATCCT & ACGCTTCAGCCTCTTC & 90 \\
\hline CD68 & ATTACCCGAGGACGACA & TCAGTTGCGAGTTGAAGA & 102 \\
\hline \multicolumn{4}{|c|}{ Persistent infection } \\
\hline Titin & TGCCTAAGACAGAACACG & GCATGAATTTCGATACCA & 160 \\
\hline Usp11 & CTTTCTCAACAACCGCTAC & ACATTCGGCACAATGGA & 135 \\
\hline \multicolumn{4}{|c|}{ Human microarray } \\
\hline Colla1 & ATACCTGCTTGATCTGTATTTGC & TCCCTCGACTCCTATGAGTTC & 167 \\
\hline Chac1 & TTGCCTGCCGAGGTTTC & GCTTCCAGGTGCTCATCC & 103 \\
\hline Colla2 & CCTAGCAACATGCCAATC & GCAAAGTTCCCAATAAG & 216 \\
\hline $\mathrm{DCN}$ & ACTCTGAAGAAGGGCAACG & TTCCCACTCCACAAAGGTAT & 257 \\
\hline TUSC3 & GCGTTACTCATCTGCTTTC & CTCTTGGGTCTGCCTTT & 238 \\
\hline CLCN3 & TGCTATGCCTCTGCG & GTGTCACCGTCTAACAAAT & 190 \\
\hline CAMK1D & CGAGGAGTCCAAGATAAT & TTCAGGAGCGACATAGC & 106 \\
\hline \multicolumn{4}{|c|}{ Mouse microarray } \\
\hline RGS16 & ACAGTTGGCTTTGGTCATAC & AATCATATTGGGCTCGTTG & 177 \\
\hline Stambp & CGTTACTTTCGTTCTGGTG & TTCTTCGGCTTTAGGGA & 213 \\
\hline HEMGN & ATGGACCTAAAGACTGCC & AAATTCTGAGGAATCCCTAC & 128 \\
\hline ZCCHC9 & CAACAGACAGCCAGGAA & ACAGCGGTAACAAATGC & 203 \\
\hline AIM1L & ATCCTCCGAACTCACCG & CCTCGTCCTCTTCTGATGC & 148 \\
\hline DOK2 & CCTCCTACGACTTTCCCACT & CACGAGCCAAGGAACCA & 112 \\
\hline NFYB & ATTACGACCACTCTGCC & CTCCCTTTCCTATGTGC & 126 \\
\hline \multicolumn{4}{|c|}{ House keeping } \\
\hline GAPDH & AAGGCCATCACCATCTTCCA & GCCAGTAGACTCCACAACATAC & 87 \\
\hline
\end{tabular}


Table S5. Gene expression validation for human and mouse microarrays

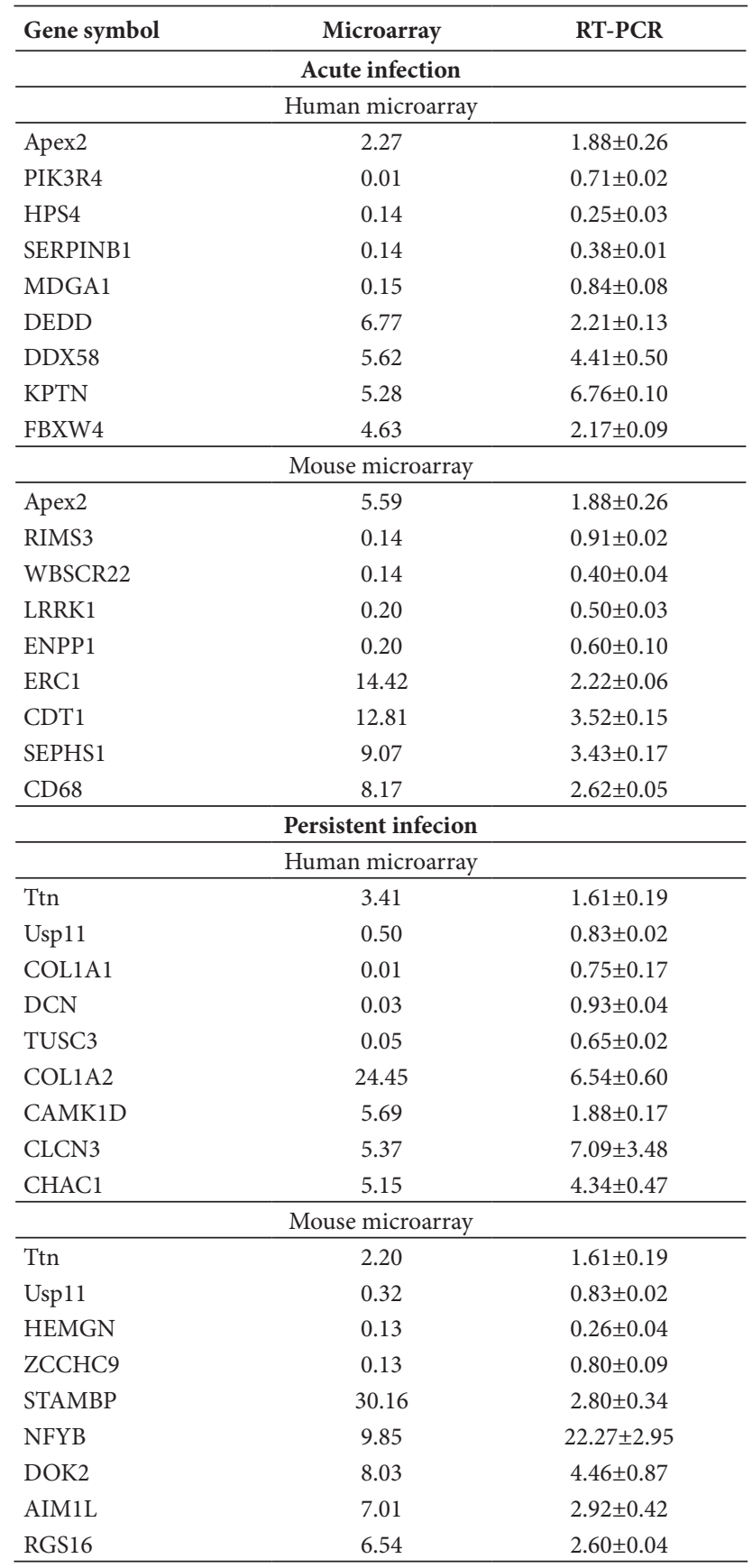

cDNA of was prepared from three independent BHK-21 cultures and used in qRT-PCR experiments using the SYBR green method and specific primers for homologous sequences of human and mouse. qRT-PCR levels of RNA for a given gene were normalized against the housekeeping gene GAPDH, and levels in FMDV infected samples expressed relative to the expression level in the corresponding Mock infected samples. Mean relative expression levels of triplication experiments using two independent RNA samples are shown. The mean expression ratios from the corresponding microarray data are also indicated. 